\title{
Dew drops on spider web appearance: a newly named pattern of IgG4 deposition in pemphigus with direct immunofluorescence
}

\author{
Marian Dmochowski ${ }^{1}$, Justyna Gornowicz-Porowska ${ }^{1}$, Monika Bowszyc-Dmochowska ${ }^{2}$ \\ ${ }^{1}$ Autoimmune Blistering Dermatoses Section, Department of Dermatology, Poznan University of Medical Sciences, Poznan, Poland \\ ${ }^{2}$ Cutaneous Histopathology and Immunopathology Section, Department of Dermatology, Poznan University of Medical Sciences, \\ Poznan, Poland
}

Adv Dermatol Allergol 2017; XXXIV (4): 295-298

DOI: https://doi.org/10.5114/ada.2017.69306

\begin{abstract}
Novel appearances in cutaneous pathology as well as mucocutaneous clinical signs are being described which indicate that this is still an attractive area for exploration. The $\mathrm{H}+\mathrm{E}$ histology terms of "decorated tomb stoning" and "undecorated tomb stoning", advocated by some pathologists, are misleading and as such should be avoided. Here, an appearance of IgG4 pemphigus deposits examined cost-effectively with direct immunofluorescence and suggested to be called "dew drops on spider web" is depicted in depth.
\end{abstract}

Key words: pemphigus, pathology, autoimmunity.

\section{Introduction to the issue of diagnosing pemphigus}

Pemphigus is a group of autoimmune blistering dermatoses. Pemphigus diseases are manifesting a surprisingly rich spectrum of clinical signs. Pemphigus diseases are potentially life threatening and require prompt clearcut diagnosis as usually aggressive immunosuppression, having serious side-effects, is needed. The key diagnostic technique is still subjective imaging direct immunofluorescence (DIF) of perilesional tissue as it detects local organ-specific autoimmunity. At present, indirect immunofluorescence (IIF) techniques, including those using biochip mosaics, are being replaced with biochemicalmolecular techniques, particularly multiparametric ELISA (developed within a framework of a multicenter project: "A European prospective study on serum antibodies against target antigens of bullous autoimmune diseases and genetic susceptibility" headquartered in Lübeck, Germany) [1].

The Tzanck cytological smears using May-GrünwaldGiemsa stain and $\mathrm{H}+\mathrm{E}$ histology have merely a supportive value as the immunological nature of pemphigus cannot be detected with them. Moreover, cutaneous non-neoplastic diseases, which are numerous, present a very limited number of patterns in $\mathrm{H}+\mathrm{E}$ histology. Ac- antholysis is a nonspecific finding [2], and can be seen in diverse dermatoses presenting disseminated, nevoid and focal arrangement; it can even be accidental. Still, $\mathrm{H}+\mathrm{E}$ histology of a lesion in question undergoing spatial-temporal evolution, if evaluated properly, can provide a hint suggesting necessary immunological investigations. $\mathrm{H}+\mathrm{E}$ histology, evaluated by a skillful pathologist, can be invaluable for differentiating pemphigus lesions from malignant ones, especially on mucous membranes.

\section{Discussion of the issue of appearances in pemphigus pathology with an emphasis on "dew drops on spider web" appearance of IgG4 deposition}

Numerous appearances in cutaneous pathology have been described aiming at easing memorization. Those appearances are pathological accompaniments to mucocutaneous clinical signs and novel appearances as well as clinical signs are being described which indicate that this is still an attractive area for exploration [3, 4]. In the context of pemphigus, we cannot agree with the $\mathrm{H}+\mathrm{E}$ histology appearance named "undecorated tomb stoning" for images seen for example in porphyrias in opposition to "decorated tomb stoning" characterizing pemphigus vulgaris and known for decades. For us, tombstones

Address for correspondence: Prof. Marian Dmochowski MD, PhD, Autoimmune Blistering Dermatoses Section, Department of Dermatology, Poznan University of Medical Sciences, 49 Przybyszewskiego St, 60-355 Poznan, Poland, phone: +48 61 8691319 , e-mail: mkdmoch@wp.pl

Received: 14.07.2016, accepted: 8.09.2016. 

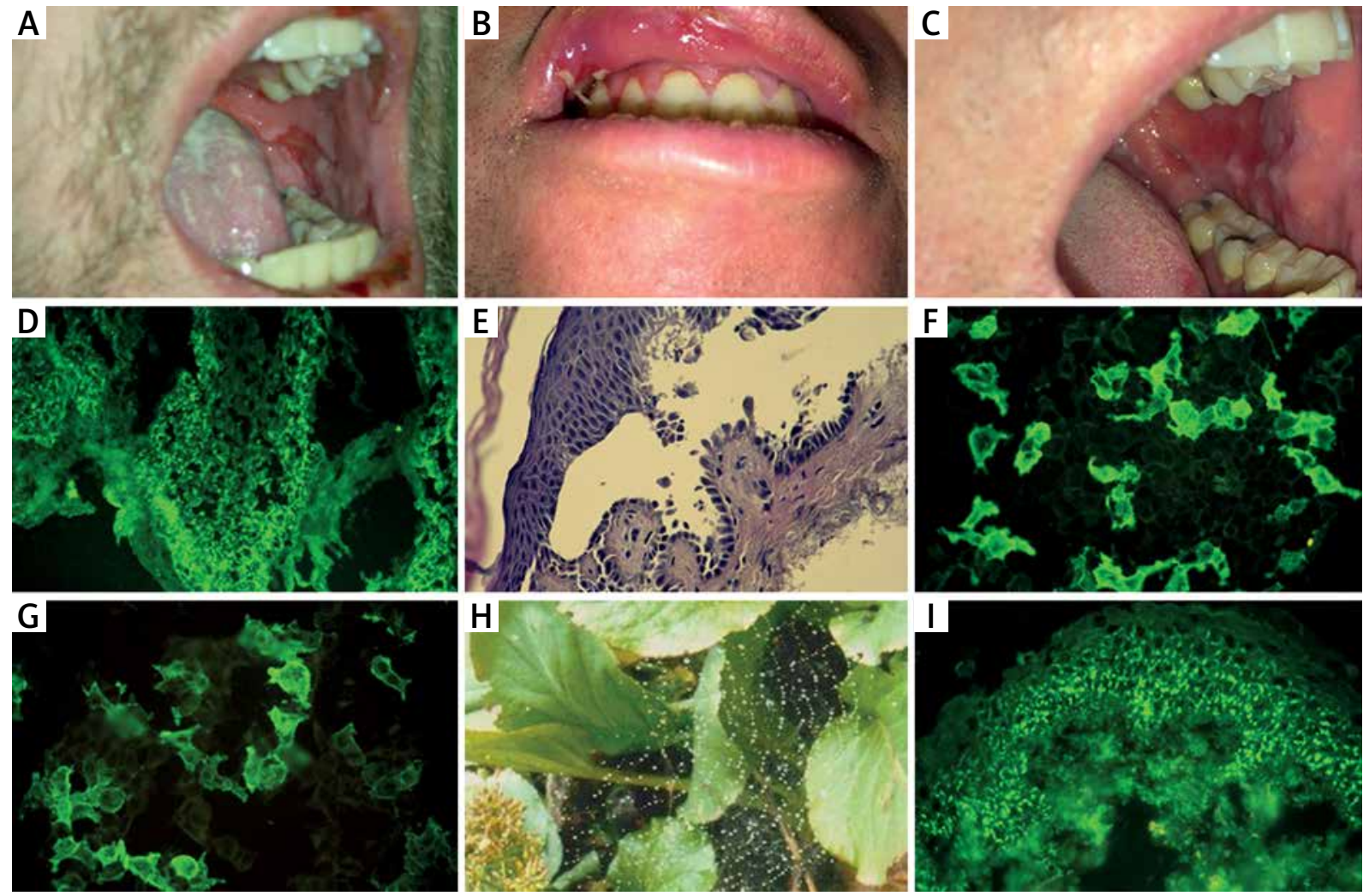

Figure 1. Male patient in his thirties with diagnosis of mucosal-dominant to and fro mucocutaneous shifting pemphigus vulgaris having initially just a slightly elevated level of serum lgG antibodies to desmoglein $3(56.86 \mathrm{RU} / \mathrm{ml})$ with stubborn course of the disease and afterwards having an elevated level of IgG antibodies to both desmoglein $1(103.908 \mathrm{RU} / \mathrm{ml})$ and desmoglein $3(184.371 \mathrm{RU} / \mathrm{ml})$ at the relapsing stage (all measurements taken using ELISA kits with cut-off values of $20 \mathrm{RU} / \mathrm{ml}$ manufactured by Euroimmun, Germany) in whom durable clinical remission could not be obtained with the range of traditional immunosuppressive therapies at first, then intravenous immunoglobulins were followed by rituximab. Initial oral presentation with erosions, crusted on vermillion border of lips, flaccid blisters and whitish exudate on the tongue causing halitosis (A), presentation with vesicles on an upper lip and desquamative gingivitis after treatment with two courses each consisting of six 5-day cycles at monthly intervals of intravenous immunoglobulins (courtesy of Anna Świrkowicz DMD) (B), presentation with an erosion of buccal mucosa a month after treatment with rituximab $1 \mathrm{~g}$ intravenously at a fortnight interval (courtesy of Anna Świrkowicz DMD) (C). The DIF of oral mucosa, performed at initial presentation using oral mucosa despite the fact that cutaneous inguinal and hand periungual lesions were present, showing just equivocal (+/-) C3, but not IgA, IgM, IgG, IgG1, IgG4, deposits (D). A row of tombstones appearance in $\mathrm{H}+\mathrm{E}$ cutaneous histology taken at the relapsing stage, in our opinion misleadingly advocated to be called "decorated tomb stoning" (E). Serum IgG (F) and IgG4 (G) pemphigus antibodies at the relapsing stage detected with IIF using human embryonal kidney HEK 293 cells transfected with extracellular and transmembranous domains of desmoglein 3 (IIF mosaic manufactured by Euroimmun, Germany). Dew drops on spider web woven on vegetation in Dmochowski's home backyard (H). Dew drops on spider web appearance of IgG4 pemphigus deposits in DIF of the perilesional skin taken at the relapsing stage (I)

mean keratinocytes, so if there are no keratinocytes in the evaluated pathological area, the term "undecorated tomb stoning" is simply misleading.

We are evaluating IgG4 deposits with DIF as their images are generally easier to interpret compared to those obtained while evaluating IgG deposits. This is particularly true, if tissue examined is fragmented and/ or crushed as it happens with mucosal specimens. Regarding DIF patterns in pemphigus, while evaluating deposits of IgG4 using a single-step approach [5], we noticed that IgG4 deposition is dotty under magnification in the range of 400-600. This dotty pattern resembles dew drops on spider web [6], so we propose calling this dew drops on spider web appearance (Figure 1) in opposition to the textbook pattern of IgG deposition known as a fish net or chicken wire appearance. We hypothesize that this dotty appearance in both extrafollicular and follicular epithelium with DIF, as it is a repetitive finding, is not a technical procedure artifact or epiphenomenon, but has true pathological meaning being pathognomonic for pemphigus.

Certain previous studies stated that a punctate or granular fluorescence may be appreciated at higher magnifications by both DIF and IIF in pemphigus in case of 




Figure 2. Pemphigus-like serum antibodies of IgG class giving granular appearance detected with IIF on monkey esophagus (A) in a middle-aged female with long-standing psoriasis taking oral methotrexate and oral cyclosporine simultaneously which induced massive oral erosions clinically suggesting mucosal-dominant pemphigus vulgaris. Using $A B$ absorbent (Euroimmun, Germany), developed with an aim to pre-absorb serum antibodies to blood groups antigens, did not affect the result when testing for IgG antibodies was done in the examined serum (B), whereas there were no pemphigus-like serum antibodies of IgG4 subclass detected both with (C) and without AB absorbent which stresses the diagnostic significance of evaluating IgG4 antibodies if one still uses IIF on monkey esophagus in the differential diagnosis of pemphigus

IgG deposits [7, 8]; however, without the molecular explanation of this phenomenon. Some researchers $[9,10]$ observed it especially in the lower layers of epidermis. It is known that dotted or granular staining of IgG does not reflect the physiological distribution of desmogleins, which should be present as a normal smooth pattern within intercellular spaces of epidermal keratinocytes. The reason for this event could be related to the clumping of desmosomal cadherins [10]. Fascinatingly, one of the possible clarifications of granular lgG staining was proposed by Oktarina et al. [10], who indicated that the lgG-induced clustering of the pemphigus autoantigens (desmogleins) underlies the granular IgG deposition in the patient skin undergoing spatial-temporal remodeling of keratinocyte surface leading to the formation of invaginations of one cell into another [11]. Moreover, this group of researchers showed that in pemphigus foliaceus and in mucocutaneous pemphigus vulgaris only desmoglein 1 clustering correlates with nonacantholytic intercellular widening between desmosomes. The sequestration of desmogleins from desmosomal components, fitting in with the desmoglein non-assembly depletion hypothesis, as well as targeting non-junctional desmogleins that are no longer available to be incorporated into desmosomes structures are processes leading this way to the disturbed assembly of desmogleins-depleted desmosomes [10]. The issue is still more complex, as regarding IIF on monkey esophagus it should be noted that pemphigus-like antibodies of IgG class misleadingly can give a granular appearance, instead of a pemphiguscharacterizing honeycomb appearance, whereas such antibodies are not detectable in IgG4 subclass and such a finding is not related to the presence in serum of ex- amined antibodies reacting with blood groups antigens expressed in the substrate tissue [12] (Figure 2). Obvious explanation might be that the substrate influences fluorescence images.

However, in light of the above, the intriguing question arises why lgG4 deposits in DIF are less continuous than IgG ones at the same magnification. The explanation may be that IgG gives more disease-nonspecific background staining involving less desmoglein-rich areas on a keratinocyte's surface whereas IgG4 is responsible for more pemphigus-specific pathology staining desmogleinrich desmosomes and extradesmosomal desmogleins. This speculation requires investigational verification as putative non-desmoglein targets might be pathogenetically relevant [13].

Putting aside detailed pathogenesis, we suggest that this dew drops on spider web appearance of IgG4 pemphigus deposits as newly described in depth here, that has already been mentioned just incidentally by us in a PubMed accessible reference [14], should be known by both laboratory workers and dermatology clinicians dealing with pemphigus. The reason for being familiar with this appearance is that evaluating lgG4 deposition in a cost-effective manner with traditional DIF is of diagnostic value, especially in undiagnosed clinically active smoldering pemphigus cases despite this treated with inadequate immunosuppression and thus having equivocal results of serum testing with ELISA techniques and DIF testing for just IgG and C3 deposition, as it enables the detection of Th2-mediated phenomena characteristic for the active stage of pemphigus [5]. It is interesting in the context of IgG4-mediated autoimmune processes in pemphigus that some patients with IgG4-related dis- 
ease experience a relapse following rituximab [15]. The relapse of this kind might be related to the survival of low memory B cells after rituximab use which was noticed in patients with pemphigus [16].

Furthermore, in drug-induced pemphigus, the pattern of immunostaining of perilesional tissue may be used as a prognostic marker since the absence of patchy staining with an antibody to desmoglein 1 and 3 is considered an indicator of favorable prognosis [17].

Finally, advanced imaging techniques such as atomicforce microscopy/scanning-force microscopy, multi-photon microscopy, super/high resolution techniques known under acronyms STED (STimulated Emission Depletion) and GSDIM/dSTORM (Ground State Depletion followed by Individual Molecule return/direct Stochastic Optical Reconstruction Microscopy) would expand our understanding of what is going on when pemphigus autoimmune recognition occurs on the surface of keratinocyte and, if adjusted to routine diagnostic use, provide novel appearances more precisely rendering reality.

\section{Conflict of interest}

The authors declare no conflict of interest.

\section{References}

1. van Beek N, Dahnrich C, Hornig N, et al. Prospective controlled studies on the routine use of a novel multivariant ELISA for the diagnosis of autoimmune bullous diseases. J Invest Dermatol 2015; 135 Suppl. 2: S33.

2. Schmieder A, Pasternack SM, Krahl D, et al. Galli-Galli disease is an acantholytic variant of Dowling-Degos disease: additional genetic evidence in a German family. J Am Acad Dermatol 2012; 66: e250-1.

3. Madke B, Kar S, Yadav N. Newly described signs in dermatology. Indian Dermatol Online J 2015; 6: 220-1.

4. Madke B, Doshi B, Khopkar U, Dongre A. Appearances in dermatopathology: the diagnostic and the deceptive. Indian J Dermatol Venereol Leprol 2013; 79: 338-48.

5. Gornowicz-Porowska J, Pietkiewicz P, Bowszyc-Dmochowska M, Dmochowski M. Immunoglobulin G4 is prevailing over immunoglobulin G1 in autoimmunity of pemphigus and bullous pemphigoid: analysis of tissue-bound antibodies in active diseases. Centr Eur J Immunol 2013; 38: 80-91.

6. Bartkiewicz P, Gornowicz-Porowska J, Bowszyc-Dmochowska $\mathrm{M}$, et al. No response to rituximab in a case of mucosaldominant to and fro mucocutaneous shifting pemphigus vulgaris previously treated with a range of more traditional anti-pemphigus therapies: clinical hint relevant to pemphigus pathogenesis. Pathogenesis of Pemphigus and Pemphigoid. Satellite Symposium to the $46^{\text {th }}$ Annual ESDR Meeting. Munich September 5-7 2016, Poster 52.

7. Mutasim DF, Adams BB. Immunofluorescence in dermatology. J Am Acad Dermatol 2001; 45: 803-22.

8. Anuradha C, Malathi N, Anandan S, Magesh K. Current concepts of immunofluorescence in oral mucocutaneous diseases. J Oral Maxillofac Pathol 2011; 15: 261-6.

9. Nousari H, Anhalt G. Pemphigus vulgaris, paraneoplastic pemphigus and pemphigus foliaceus. In: Diagnostic Im- munohistochemistry of the Skin. $1^{\text {st }}$ ed. Kanitakis J, Vassileva S, Woodley D (eds). Chapman \& Hall, London 1998; 74-83.

10. Oktarina DA, van der Wier G, Diercks GF, et al. IgG-induced clustering of desmogleins 1 and 3 in skin of patients with pemphigus fits with the desmoglein nonassembly depletion hypothesis. Br J Dermatol 2011; 165: 552-62.

11. Sokol E, Nijenhuis M, Kramer D, et al. Pemphigus vulgaris autoantibodies cause invaginations of one cell into another. Pathogenesis of Pemphigus and Pemphigoid. Satellite Symposium to the $46^{\text {th }}$ Annual ESDR Meeting. Munich September 5-7 2016, Poster 29.

12. Gornowicz-Porowska J, Świrkowicz A, Bartkiewicz P, et al. Wstępna ocena użyteczności absorbentu AB w diagnozowaniu pęcherzycy metodą immunofluorescencji pośredniej na przełyku małpim. Prz Dermatol 2016; 103 Suppl. 1: S220-1.

13. Ahmed AR, Carrozzo M, Caux F, et al. Monopathogenic vs. multipathogenic explanations of pemphigus pathophysiology. Exp Dermatol 2016 Jun 15. doi: 10.1111/exd.13106. [Epub ahead of print]

14. Pietkiewicz P, Gornowicz-Porowska J, Bowszyc-Dmochowska M, Dmochowski M. A retrospective study of antihypertensives in pemphigus: a still unchartered odyssey particularly between thiols, amides and phenols. Arch Med Sci 2015; 11: 1021-7.

15. Wallace ZS, Mattoo H, Mahajan VS, et al. Predictors of disease relapse in IgG4-related disease following rituximab. Rheumatology (Oxford) 2016; 55: 1000-8.

16. Stamey C, Pasieka HB, Anhalt G, et al. The identification of CD20 negative/low memory B cells in pemphigus patients. The Society for Investigative Dermatology 2015 Annual Meeting, Atlanta, GA, May 6-9 2015, Late breaking abstract LB739.

17. Maruani A, Machet MC, Carlotti A, et al. Immunostaining with antibodies to desmoglein provides the diagnosis of drug-induced pemphigus and allows prediction of outcome. Am J Clin Pathol 2008; 130: 369-74. 